\title{
Die Europäische Staatsanwaltschaft - Ein wirksames Instrument zur Bekämpfung von Korruption?
}

Gastautor

2019-10-31T16:27:35

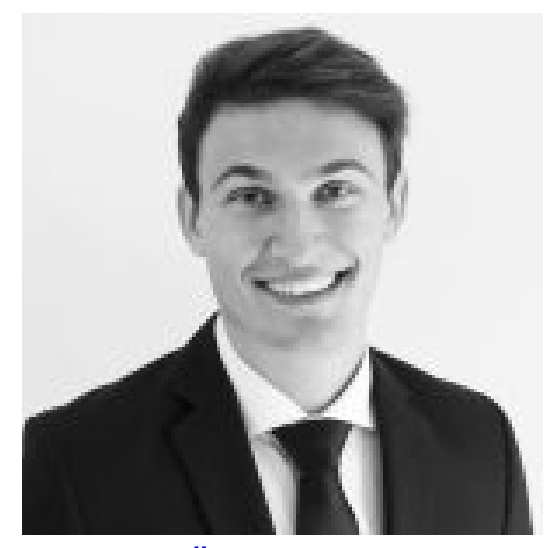

DAVID LÖFFLER

von

Nach einer langen Diskussion konnten sich das Europäische Parlament und der Rat auf die rumänische Juristin Laura Kövesi als Leiterin der Europäischen Staatsanwaltschaft einigen. Die neue unabhängige Strafermittlungsbehörde der EU wird ihre Tätigkeit voraussichtlich Ende 2020 aufnehmen. Mit der Europäischen Staatsanwaltschaft soll eine Einrichtung geschaffen werden, welche die effiziente Bekämpfung von Straftaten gegen die finanziellen Interessen der EU gewährleistet. Kann sie diesem Ziel auch gerecht werden?

\section{Errichtung im Wege der Verstärkten Zusammenarbeit}

Die Möglichkeit zur Schaffung einer Europäischen Staatsanwaltschaft wurde mit dem Vertrag von Lissabon in Art. 86 AEUV primärrechtlich verankert. Gemäß Abs. 1 leg. cit. kann der Rat mit Zustimmung des Europäischen Parlaments mittels Verordnung eine Europäische Staatsanwaltschaft zur Bekämpfung von Straftaten zum Nachteil der finanziellen Interessen der EU einrichten. Im Rat kam jedoch die erforderliche Einstimmigkeit nicht zustande. Deshalb wurde vom Großteil der Mitgliedstaaten von der Möglichkeit der Verstärkten Zusammenarbeit Gebrauch gemacht und die Verordnung (EU) 2017/1939 (EUStA-VO) erlassen, welche am 20. November 2017 in Kraft trat. Nach derzeitigem Stand sind 22 Mitgliedstaaten der Europäischen Staatsanwaltschaft beigetreten. Dänemark, Irland, Polen, Schweden, Ungarn und das demnächst aus der EU ausscheidende Vereinigte Königreich nehmen nicht teil. In diesen Staaten wird es weiterhin ausschließlich Sache der nationalen Ermittlungsbehörden sein, Straftaten zulasten des EU-Haushalts zu verfolgen. 


\section{Aufbau der Europäischen Staatsanwaltschaft}

Die Europäische Staatsanwaltschaft setzt sich aus einer zentralen Dienststelle mit Sitz in Luxemburg und den Delegierten Europäischen Staatsanwälten zusammen (Art. 8 EUStA-VO). Diese Aufgliederung in eine zentrale und dezentrale Ebene stellt eine Kompromisslösung dar, da einige Mitgliedstaaten in einer rein zentral aufgebauten Europäischen Staatsanwaltschaft einen zu weit gehenden Eingriff in ihre nationale Souveränität sahen. Dennoch agieren alle Mitglieder der Europäischen Staatsanwaltschaft - auch die Delegierten Europäischen Staatsanwälte - bei ihren Ermittlungen weisungsfrei gegenüber den Mitgliedstaaten und den Unionsorganen (Art. 6 Abs. 1 EUStA-VO).

Die Leitung der Europäischen Staatsanwaltschaft obliegt dem Europäischen Generalstaatsanwalt (Art. 11 Abs. 1 EUStA-VO). Dieser wird gemäß Art. 14 Abs. 1 EUStA-VO vom Europäischen Parlament und dem Rat ,in gegenseitigem Einvernehmen" ernannt. Im Bewerbungsverfahren setzte sich schlussendlich die bevorzugte Kandidatin des Europäischen Parlaments, Laura Kövesi, durch. Die 46-jährige Juristin leitete von 2013 bis 2018 die Nationale Antikorruptionsbehörde Rumäniens. In dieser Zeit machte sie sich als „Korruptionsjägerin“ einen Namen, da einige bekannte Politiker und Unternehmer im Zuge ihrer Amtszeit wegen Korruptionsdelikten angeklagt und verurteilt wurden.

In der zentralen Dienststelle befinden sich die Europäischen Staatsanwälte sowie die Ständigen Kammern. Vom Rat wird aus jedem Mitgliedstaat ein Europäischer Staatsanwalt ernannt (Art. 16 Abs. 3 EUStA-VO). Die Ständigen Kammern bestehen aus einem Vorsitzenden und zwei Europäischen Staatsanwälten. Den Vorsitz hat der Europäische Generalstaatsanwalt oder einer seiner Stellvertreter inne (Art. 10 Abs. 1 EUStA-VO). Sowohl die Europäischen Staatsanwälte als auch die Ständigen Kammern sind für die Beaufsichtigung der Ermittlungsverfahren zuständig (Art. 10 Abs. 2, Art. 12 Abs. 1 EUStA-VO). Die Delegierten Europäischen Staatsanwälte führen hingegen die Ermittlungen in den Mitgliedstaaten durch und haben dabei dieselben Befugnisse wie nationale Staatsanwälte. Sie unterliegen den Weisungen einer Ständigen Kammer und des beaufsichtigenden Europäischen Staatsanwalts (Art. 13 Abs. 1 EUStA-VO).

\section{Zuständigkeitsbereich}

Gemäß Art. 22 EUStA-VO ist die Europäische Staatsanwaltschaft vor allem für die Verfolgung von Straftaten zuständig, welche sich aus der Richtlinie (EU) 2017/1371 (PIF-RL) ergeben. Dazu zählen etwa Betrug, Geldwäsche oder Bestechung zum Nachteil der finanziellen Interessen der EU. In diesem Bereich besteht durchaus Handlungsbedarf: Laut Kommission belief sich das durch Betrug verursachte Schadensvolumen zulasten des EU-Haushalts allein im Jahr 2018 auf ca. 1,2 Milliarden Euro. Die Dunkelziffer wird weitaus höher geschätzt.

Derzeit sind einzig die nationalen Behörden befugt, bei Straftaten gegen die finanziellen Interessen der EU zu ermitteln. Diese können natürlich lediglich innerhalb des jeweiligen nationalen Hoheitsgebiets tätig werden, weshalb sich die 
Strafverfolgung bei grenzüberschreitenden Fällen oftmals als schwierig gestaltet. Mit dem Europäischen Amt für Betrugsbekämpfung (OLAF) besteht zwar eine unionsrechtliche Einrichtung, welche bei rechtswidrigen Handlungen zum Nachteil des EU-Haushalts ermittelt, jedoch sind dessen Befugnisse auf die Durchführung verwaltungsrechtlicher Untersuchungen beschränkt.

Die Mitgliedstaaten verfolgen Korruption zulasten des EU-Haushalts nicht immer mit letzter Konsequenz, vor allem, wenn kein Schaden für die eigene Wirtschaft entsteht. So wurde etwa im März 2018 ein Vertragsverletzungsverfahren gegen das Vereinigte Königreich eingeleitet, da dieses laut Kommission seit Jahren keine angemessenen Maßnahmen gegen die betrügerische Umgehung von Zöllen bei der Einfuhr von Bekleidung und Schuhen aus China ergreift. Die geringe Motivation des Vereinigten Königreichs zum Handeln lässt sich wohl damit erklären, dass die an den EU-Außengrenzen eingehobenen Zölle abzüglich der Erhebungskosten zur Gänze dem EU-Haushalt zugutekommen. Der Fall ist mittlerweile beim EuGH anhängig.

\section{Ablauf des Ermittlungsverfahrens}

Besteht der Verdacht einer Straftat, welche in den Zuständigkeitsbereich der Europäischen Staatsanwaltschaft fällt, leitet einer der Delegierten Europäischen Staatsanwälte im Mitgliedstaat, in dem sich die Tat ereignet hat, das Ermittlungsverfahren ein (Art. 26 Abs. 1 EUStA-VO). Bei grenzüberschreitenden Fällen kommt es für die Zuständigkeit hinsichtlich des Ermittlungsverfahrens darauf an, in welchem Mitgliedstaat der Schwerpunkt der Straftat liegt (Art. 26 Abs. 4 EUStA-VO).

Der zuständige Delegierte Europäische Staatsanwalt führt die Ermittlungen entweder selbst durch oder weist die nationale Strafverfolgungsbehörde an, die erforderlichen Maßnahmen zu treffen (Art. 28 Abs. 1 EUStA-VO). Daneben kann die Europäische Staatsanwaltschaft auch das OLAF ersuchen, Informationen bereitzustellen und verwaltungsrechtliche Untersuchungen durchzuführen (Art. 101 Abs. 3 EUStA-VO). Nach Abschluss des Ermittlungsverfahrens berichtet der Delegierte Europäische Staatsanwalt dem beaufsichtigenden Europäischen Staatsanwalt, ob Anklage vor einem Gericht des betroffenen Mitgliedstaats erhoben werden soll. Dieser leitet den Bericht an die Ständige Kammer weiter, welche dann über die tatsächliche Anklageerhebung beschließt (Art. 35 Abs. 1, 36 Abs. 1 EUStA-VO).

\section{Fazit: Kooperationsbereitschaft der Mitgliedstaaten als Schlüssel zum Erfolg}

Bisher war der Schutz der finanziellen Interessen der EU nicht unbedingt ein wichtiges Anliegen für alle Mitgliedstaaten. Die Schaffung einer unabhängigen Ermittlungsbehörde, welche sachlich umfassend und grenzüberschreitend tätig werden kann, stellt daher einen wichtigen Schritt bei der Bekämpfung von Straftaten zulasten des EU-Haushalts dar. Mit Laura Kövesi steht eine erfahrene Juristin im Kampf gegen Korruption an der Spitze der Europäischen Staatsanwaltschaft. 
Ihre Ernennung stimmt jedenfalls positiv, dass die Behörde auch die nötige Durchschlagskraft haben wird.

Die Organisationsstruktur der Europäischen Staatsanwaltschaft wirkt auf den ersten Blick etwas komplex. Unverständlich ist vor allem, warum neben einer Ständigen Kammer auch noch ein einzelner Europäischer Staatsanwalt für die Aufsicht im Ermittlungsverfahren zuständig ist und dem ermittelnden Delegierten Europäischen Staatsanwalt direkt Weisungen erteilen kann. Dies könnte zu Unklarheiten führen und das Ermittlungsverfahren unnötig in die Länge ziehen.

Da die Europäische Staatsanwaltschaft über keinen umfangreichen Behördenapparat verfügt, wird der Erfolg ihrer Ermittlungen letztlich von der Unterstützung der Mitgliedstaaten abhängen. Die Einschaltung von Delegierten Europäischen Staatsanwälten ist hierbei auf jeden Fall von Vorteil. Sie kennen die Eigenheiten der nationalen Rechtsordnungen und können durch ihre Erfahrung für eine gut funktionierende Zusammenarbeit mit den nationalen Ermittlungsbehörden sorgen. Das Konzept einer zentralen und dezentralen Ebene hat daher durchaus seine Vorzüge.

Bedauerlicherweise beteiligen sich nicht alle Mitgliedstaaten an der Europäischen Staatsanwaltschaft. Dies birgt die Gefahr einer künftigen Zweiklassengesellschaft bei der Verfolgung von Straftaten zulasten der finanziellen Interessen der EU. Insbesondere sind der Europäischen Staatsanwaltschaft bei grenzüberschreitenden Fällen, welche nicht teilnehmende Mitgliedstaaten betreffen, gewissermaßen die Hände gebunden. Durchaus problematisch ist zudem der dynamische Verweis in Art. 22 EUStA-VO auf die PIF-RL hinsichtlich der sachlichen Zuständigkeit der Europäischen Staatsanwaltschaft. Diese Richtlinie wurde von allen Mitgliedstaaten im ordentlichen Gesetzgebungsverfahren mit qualifizierter Mehrheit erlassen. Bei einer etwaigen Änderung der PIF-RL haben die nicht teilnehmenden Mitgliedstaaten somit die Möglichkeit, über die Zuständigkeit der Europäischen Staatsanwaltschaft mitzubestimmen. Hier werden die Probleme deutlich, welche im Rahmen der Verstärkten Zusammenarbeit mitunter auftreten können. Aus diesen Gründen bleibt zu hoffen, dass die restlichen Mitgliedstaaten zu einem späteren Zeitpunkt doch noch für eine Teilnahme an der Europäischen Staatsanwaltschaft gewonnen werden können.

Zitiervorschlag: Löffler, Die Europäische Staatsanwaltschaft - Ein wirksames Instrument zur Bekämpfung von Korruption?, JuWissBlog Nr. 96/2019 v. 31.10.2019, https://www.juwiss.de/96-2019/.

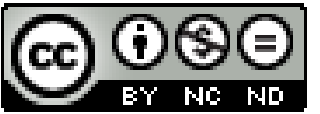

Dieses Werk ist lizenziert unter einer Creative Commons Namensnennung - Nicht kommerziell - Keine Bearbeitungen 4.0 International Lizenz. 
(cc) EY-NC-ND

-5 - 